\title{
Coexistence of long-range orders in low-dimensional systems ${ }^{\star}$
}

\author{
Sudhakar Yarlagadda ${ }^{1, a}$ and Peter B. Littlewood ${ }^{2,3}$ \\ ${ }^{1}$ Saha Institute of Nuclear Physics, Kolkata, India \\ 2 Argonne National Laboratory, Argonne, IL, USA \\ 3 James Franck Institute, University of Chicago, Chicago, IL, USA
}

Received 30 May 2018

Published online 17 September 2018

(C) EDP Sciences / Società Italiana di Fisica / Springer-Verlag GmbH Germany, part of Springer Nature, 2018

Strongly correlated condensed matter systems are responsible for some of the interesting broken symmetry phases of matter: this ranges from insulating structures such as ferroelectrics and charge density waves, orbital order, magnetism, and superconductivity. Correspondingly, transition metals and their oxides have practical utility for their functional character. As well as attempting to understand the plethora of phases, the role of experiment and theory is also to control them, often with doping, but also with external fields (electromagnetic and stress), and subtly by manipulating through interfacial interactions, atomic size effects and otherwise. A feature of these correlated systems is that the free energy differences between phases can be small so competition is evident, but more subtly coexistence can be favored by the competition of interactions. This coexistence may be homogeneous, when the orders are compatible, or inhomogeneous leading to incommensurate or disordered structures.

In this collection of articles, leading researchers have contributed to this rapidly growing field, of coexistence, competition and long-range order. The scene is set by some new experimental systems: Haines and Saxena [1] present the discovery of an insulator to metal transition in $\mathrm{FePS}_{3}$ under pressure; and in a different system $\mathrm{Cs}_{2} \mathrm{CuCl}_{4}$ which is close to a spin liquid ground state, the irreversible appearance of a new magnetically-ordered state with application and release of pressure. Grant et al. [2] have found a new way to manipulate coexistence behavior in manganites, in particular to control coercive fields in thin films. New topics addressed by theory include the possibility of stabilizing a finite momentum-pairing state in the crystalline topological insulator PbSnTe [3], and competing structural and magnetic instabilities in the Fe-based superconductors [4]. Baskaran [5] provides

\footnotetext{
* Contribution to the Topical Issue "Coexistence of LongRange Orders in Low-dimensional Systems", edited by Sudhakar Yarlagadda and Peter B. Littlewood.

${ }^{a}$ e-mail: y.sudhakar@saha.ac.in
}

a review of valence bond states in Mott physics and their description in terms of emerging gauge fields.

There are some still beguiling problems in model quantum spin systems that are increasingly amenable to numerical solution: the phase diagram of ShastrySutherland type models, with evidence from Monte Carlo simulations of the existence of a supersolid phase [6]; a resolution of the paradox of melting of three-sublattice order in a triangular lattice antiferromagnet [7]. We gain great insight by models where exact solutions are available, and Nakamura and Nishimoto [8] provide a review of the construction of exact ground states of extended Hubbard like models.

Many physical systems show the results of direct competition between lattice effects, or spin-driven magnetism, and Coulomb coupling. Hohenadler and Fehske [9] review the results of $1 \mathrm{D}$ half-filled fermion lattices, taking into account coupling to the lattice with quantum phonons, and competition mediated also by Coulomb interactions in a range of models. Ghosh et al. [10] study one of these models - the Hubbard-Holstein model - in two dimensions, providing strong evidence for the elusive supersolid. The other classic competition in 2D systems, between antiferromagnetism and superconductivity is studied by Kadge and Majumdar [11], in a Hubbard-like model at half-filling but intermediate coupling. Related physics is attacked by Abdelwahab and Jeckelman [12] in an asymmetric Hubbard ladder that has interacting strong- and weak-coupling channels.

The range of materials, and models, has now expanded far beyond the classic simplified Hubbard models, but the new systems provide more than just richness and complexity. There are entirely new phenomena under the robust control of topology, and new experimental avenues to tuning via pressure, anisotropic strain, and morphology. The theorists have developed methods for highly accurate solutions on large systems so that quite precise statements can now be made. 


\section{References}

1. C.R.S. Haines, S.S. Saxena, Eur. Phys. J. B 91, 196 (2018)

2. D. Grant, M. Ryan, A. Biswas, Eur. Phys. J. B 91, 197 (2018)

3. S. Kundu, V. Tripathi, Eur. Phys. J. B 91, 198 (2018)

4. K. Ali, K. Maiti, Eur. Phys. J. B 91, 199 (2018)

5. G. Baskaran, Eur. Phys. J. B 91, 200 (2018)

6. K. Wierschem, Z. Zhang, A. Wibawa, P. Sengupta, Eur. Phys. J. B 91, 201 (2018)
7. D. Heidarian, K. Damle, Eur. Phys. J. B 91, 202 (2018)

8. M. Nakamura, S. Nishimoto, Eur. Phys. J. B 91, 203 (2018)

9. M. Hohenadler, H. Fehske, Eur. Phys. J. B 91, 204 (2018)

10. A. Ghosh, S. Kar, S. Yarlagadda, Eur. Phys. J. B 91, 205 (2018)

11. S. Kadge, P. Majumdar, Eur. Phys. J. B 91, 206 (2018)

12. A. Abdelwahab, E. Jeckelmann, Eur. Phys. J. B 91, 207 (2018) 